\title{
Brightness and size transposition by rhesus monkeys
}

\author{
ROBERT PASNAK and STEVEN L. KURTZ \\ George Mason University, Fairfax, Virginia
}

\begin{abstract}
Two experimentally naive adult rhesus monkeys were trained on a VR4 schedule to make brightness discriminations. Transposition tests showed that these monkeys discriminated on the basis of absolute rather than relative brightness. These monkeys and a third (naive) one were trained by the same method to make size discriminations. Size transposition tests showed that the monkeys responded to absolute rather than relative size. The monkey with no training on brightness discrimination had much broader generalization gradients, replicating the results of other investigations of size transposition.
\end{abstract}

If rhesus monkeys are trained to discriminate between one pair of stimuli and are then confronted with novel stimulus pairs, they appear to discriminate on the basis of the relation between the stimuli. This type of transfer is called transposition (Reese, 1968). The purpose of the present research was to determine the basis for this transfer.

Responses that appear to be based on the relation between the stimuli may in fact be based on absolute qualities of the stimuli (Spence, 1937). If generalization gradients were based on absolute stimulus qualities, they would produce responding that paralleled the relation between the stimuli, even though not based on that relation, over most of the stimulus continuum. An important exception would occur for stimulus pairs at some positions on the positive side of the continuum (i.e., the side demarcated by the positive stimulus in the original pair). Reversals, or choices opposite to those predicted on the relation between stimuli, should occur on the positive side of the continuum, especially on far tests (i.e., tests involving pairs of stimuli that do not include either of the original training stimuli). For near tests (i.e., tests involving stimulus pairs that include one of the original discriminanda) on either side of the continuum or for far tests on the negative side, reversals should not occur even when the discrimination is based on absolute stimulus qualities, unless the generalization gradients are relatively narrow.

Random responding could also occur at either end of the stimulus continuum, whether the original discrimination was based on relational or absolute stimulus characteristics. Random responding can be interpreted as an absence of stimulus control (i.e., that the absolute or relational characteristics of the original stimuli no longer govern responding) and is not a decisive indication of the basis for the systematic responses made to other pairs of stimuli. Although test and training stimuli and test and

Special thanks are extended to Mortimer Mishkin for gracious criticism and advice. Reprint requests should be sent to Robert Pasnak, Department of Psychology, George Mason University, 4400 University Drive, Fairfax, VA 22030. training methods have varied widely, other researchers have found that rhesus monkeys' responses to both near and far tests on the size continuum have paralleled the relation between the stimuli, although random responding is sometimes observed (Ackles, R. R. Zimmerman, Manning, \& Kazarian, 1974; Bagshaw \& Pribram, 1965; Butter \& Doehrman, 1968; Harmon, Strong, \& Pasnak, 1982; Spence, 1937; R. W. Zimmerman, 1968). Such results seem to justify Schwartz's (1984) conclusion that tests of transposition itself support the view that responses are based on the relation between the stimuli.

However, at least some monkeys in one of the groups trained in brightness discrimination by Ackles et al. (1974) made far test choices that represented a statistically significant reversal of the relation. This result is potentially important, since it is virtually the only outcome of a transposition experiment with nonhuman primates that clearly supports the hypothesis that their responses are based on absolute qualities of the stimulus. Yet this result is opposite to that obtained from the other group of monkeys trained in brightness discrimination by Ackles et al., and from those trained on a similar discrimination by $R$. W. Zimmerman (1968). Hence, the present research was designed to measure the transposition of brightness discrimination under the conditions used by Harmon et al. (1982), which maximized stimulus control and produced responses that quite reliably paralleled the relation between the training stimuli.

\section{EXPERIMENT 1}

\section{Method}

Subjects. The subjects were two experimentally naive 7-year-old female rhesus monkeys (Macaca mulatta), identified as Lilly and Penny.

Apparatus. The apparatus was a portable Wisconsin General Test Apparatus (WGTA) having a $1.52 \times 1.22 \mathrm{~m}$ brown masonite baffle with a $53.3 \times 30.5 \mathrm{~cm}$ sliding door mounted along the long axis of a $71.1 \times 45.7 \mathrm{~cm}$ platform. Stimuli were presented on a $25.4 \times 20.3 \mathrm{~cm}$ sliding tray when the sliding door was raised. The monkey had to move the correct stimulus backward on its hinged mounting to obtain the food reward located in a well beneath it. 
The stimuli were $7.6 \times 5.1 \mathrm{~cm}$ rectangles of Munsell color paper protected with washable transparent plastic. The Munsell color paper was coded in whole-number increments from 2 (darkest) to 9 (brightest).

Lilly was trained with the 6 stimulus (bright positive) and the 4 stimulus (dark negative). She was tested with three novel stimulus pairs (positive listed last): 2 versus 4,6 versus 8 , and 7 versus 9 . The first two pairs constitute near tests on the negative and positive sides of the continuum; the last pair is a far test on the positive side of the continuum for Lilly.

Penny was trained to discriminate the 5 stimulus (positive) from the 7 stimulus (negative); hence, she was trained dark positive. Test stimuli were 7 versus 9 and 3 versus 5 (near tests) and 2 versus 4 (a far test on the positive side of the continuum).

Procedure. Initially the monkeys were trained to discriminate between two simultaneously presented stimuli, with selection of the positive stimulus (6 for Lilly, 5 for Penny) always rewarded with a small bit of fruit. Left or right positioning of the stimuli was randomized via a schedule of restricted randomization. The schedule for rewards for correct responses was gradually raised to a VR4 schedule. The monkeys were overtrained for 300 trials after reaching a 98\% (49/50) criterion at each VR level before advancing to the next level.

The three novel pairs of test stimuli described previously for each monkey, as well as the pair of stimuli used for training, were employed in the transposition tests (see Table 1). The pair of training stimuli was presented 45 times, and the three novel pairs of stimuli were presented 15 times each. The pair of training stimuli was alternated with the novel stimulus pairs, which were presented in a (restricted) random order. During testing a VR4 reward schedule was used with correct selection as a criterion for reward on the training stimulus pairs only. Selection of either stimulus from the test stimulus pairs was rewarded if indicated by the VR4 schedule.

\section{Results}

The results, shown in Table 1 , indicate that the responses were based on absolute properties of the stimuli, not on the relation between the stimuli. The great majority of choices for both monkeys on the far tests actually represent reversals of the relation between the stimuli. These choices are easily explained by generalization curves based on the absolute characteristics of the stimuli.

For the novel pair of stimuli, which constituted a near test on the negative side of the continuum, both monkeys tended to choose the stimulus most like the positive training stimulus. This tendency is significant $(z=2.01$,

Table 1

Responses on Tests of Brightness Transposition

\begin{tabular}{|c|c|c|c|}
\hline & \multirow{2}{*}{$\begin{array}{l}\text { Munsell Paper } \\
\text { Numbers }\end{array}$} & \multicolumn{2}{|c|}{$\begin{array}{l}\text { Choices of } \\
\text { Stimulus* }\end{array}$} \\
\hline & & Frequency & $\overline{\%}$ \\
\hline \multicolumn{4}{|c|}{ Lilly } \\
\hline Near Test Stimuli & 2 vs. 4 & 11 of 15 & 73 \\
\hline Training Stimuli & $4(-)$ vs. $6(+)$ & 45 of 45 & $100 \dagger$ \\
\hline Near Test Stimuli & 6 vs. 8 & 14 of 15 & $93+$ \\
\hline Far Test Stimuli & 7 vs. 9 & 4 of 15 & 27 \\
\hline \multicolumn{4}{|c|}{ Penny } \\
\hline Near Test Stimuli & 7 vs. 9 & 10 of 15 & 67 \\
\hline Training Stimuli & $5(+)$ vs. $7(-)$ & 38 of 45 & $84 \dagger$ \\
\hline Near Test Stimuli & 3 vs. 5 & 2 of 15 & $13 \dagger$ \\
\hline Far Test Stimuli & 2 vs. 4 & 1 of 15 & $7 \dagger$ \\
\hline
\end{tabular}

Note-Stimulus 2 is the darkest, 9 the brightest. * *illy was trained that bright was positive; Penny was trained that dark was positive. Choices of stimulus indicate the frequency with which Lilly chose bright and Penny chose dark. $\quad t p<.05$. $p<.05)$ if the data from both monkeys are pooled. This result would be expected, regardless of whether responses were based on either absolute or relational properties of the stimuli. However, Penny (trained dark positive) consistently chose the brighter of the two stimuli on both the near and far tests on the positive end of the continuum. These reversals are statistically significant according to direct calculations of binomial probabilities $(p<.05)$. Lilly (trained bright positive) tended to choose the brighter stimulus on the near test on the positive side of the continuum $(p<.05)$. This result is similar to that Schwartzbaum and Pribram (1960) obtained from rhesus monkeys on a near test of brightness transposition on the positive side of the continuum, and is in sharp contrast to Penny's performance on the comparable test. Lilly, therefore, may have exhibited a much broader generalization gradient than Penny.

Lilly chose the darker stimulus most often on the far test at the positive end of the continuum. Although the reversal falls (just) short of statistical significance, the performance is similar to that of the other monkey and again can be explained by positing generalization curves that are broader.

\section{Discussion}

These results seem to refute the interpretation of size transposition advanced by Harmon et al. (1982). Those researchers found that the response of rhesus monkeys to pairs of stimuli on the size continuum paralleled the relation between the stimuli for all test pairs, and that the percentage of responses doing so was high for all stimulus test pairs. They concluded that the monkeys responded to the relation between the stimuli. However, the monkeys in that study were trained by the same method that we use in this study, so the basis for responding should be the same in both. Since the decisions of Penny and Lilly could not have been made on the basis of the relation between the stimuli, it seems likely that the responses of Harmon et al.'s (1982) monkeys were in fact based on absolute properties of the stimuli, mediated by broad generalization curves. This is probably also true of those monkeys whose responses paralleled the relation between the stimuli in other studies of size transposition (Ackles et al., 1974; Bagshaw \& Pribram, 1965; Butter \& Doehrman, 1968; R. W. Zimmerman, 1968).

An alternative is that rhesus monkeys respond differently to size discrimination problems than to brightness discrimination problems, simply abstracting size relations more easily than brightness relations. Although there is no convincing rationale for such a difference, it is true that one of two groups of monkeys trained by Ackles et al. (1974) on a brightness discrimination task showed reversals on far tests, a result conspicuous in its absence on tests of size transposition. In Experiment 2, we taught Penny and Lilly size discrimination and tested them for size transposition in the manner employed by Harmon et al. (1982). The question was whether the monkeys would respond to size in the same way as they responded to brightness, or whether the results obtained by Harmon et al. for size transposition would be replicated.

\section{EXPERIMENT 2}

\section{Method}

Subjects and Apparatus. The same WGTA and monkeys, plus an experimentally naive 5-year-old male (Burt) were used in this experiment. The stimuli were sky-blue rectangular wooden blocks, $1.59 \mathrm{~cm}$ thick and $6.03 \mathrm{~cm}$ wide, varying in height from 2.54 to $50.80 \mathrm{~cm}$ in $2.54-\mathrm{cm}$ increments.

Procedure. Training was identical to that employed in Experiment 1, except that the negative training stimulus for Lilly and Penny was 
$12.70 \mathrm{~cm}$ and the positive one was $17.78 \mathrm{~cm}$. These stimuli have the same heights as those used by Harmon et al. (1982). For Burt the negative training stimulus was $22.86 \mathrm{~cm}$ and the positive one $27.94 \mathrm{~cm}$. These larger stimuli were employed to broaden the range of possible test pairs on the negative (small) side of the size continuum.

As in Experiment 1, subjects were initially trained to a $98 \%$ criterion (49/50) on a continuous reinforcement schedule, and were gradually raised to a VR4 schedule.

Testing was done on 5 consecutive days, and the food the monkeys earned on the test was all that they received during this period. The stimulus pairs for all monkeys are shown in Table 2. For each monkey, the pair of training stimuli was alternated with the novel stimulus pairs, which were presented in a restricted random order. Rewards were presented on a VR4 schedule, with correct selection a criterion for reward on the training stimulus pairs only. Selection of either stimulus from the test stimulus pairs was rewarded if indicated by the VR4 schedule.

\section{Results}

Both Penny and Lilly chose the larger stimulus-paralleling the relation between the training stimuli-on the near and far tests on the negative side of the stimulus con- tinuum (see Table 2). For Lilly, but not Penny, the choices became random at the very end of this side of the continuum. On the positive side of the continuum, both monkeys chose the smaller stimulus more often for some stimulus pairs, in opposition to the relation between the training stimuli. For both Penny and Lilly, those reversals that were consistent enough to be statistically significant occurred on near tests.

Three of the near tests were unusual in that they involved pairing the negative $(12.70 \mathrm{~cm})$ training stimulus with stimuli from the positive side of the continuum $(22.86 \mathrm{~cm}, 27.94 \mathrm{~cm}$, and $33.02 \mathrm{~cm}$, respectively). Penny and Lilly differed significantly in their responses to the latter two pairs $(z=2.23$ and 2.61 , respectively; $p<.05)$. Lilly's responses paralleled the relation between the training stimuli for all three pairs, whereas Penny's choices constituted reversals of this relation for the last two.

The responses of these 2 monkeys on the far tests never differed by more than 2.5 choices from the mean (7.5)

Table 2

Responses on Test of Size Transposition

\begin{tabular}{|c|c|c|c|c|c|c|}
\hline \multirow{3}{*}{$\begin{array}{c}\text { Stimulus Pair } \\
\text { (in } \mathrm{cm} \text { ) }\end{array}$} & \multicolumn{6}{|c|}{ Choices of Larger Stimulus } \\
\hline & \multicolumn{2}{|c|}{ Lilly } & \multicolumn{2}{|c|}{ Penny* } & \multicolumn{2}{|c|}{ Burt } \\
\hline & Frequency & $\%$ & Frequency & $\%$ & Frequency & $\%$ \\
\hline 2.54 vs. 5.08 & 8 of 15 & 53 & 11 of 11 & $100 t$ & & \\
\hline 2.54 vs. 7.62 & 11 of 15 & 73 & 11 of 11 & $100 \dagger$ & 6 of 12 & 50 \\
\hline 2.54 vs. 10.16 & 15 of 15 & $100 \dagger$ & 14 of 14 & $100 \dagger$ & 10 of 12 & $83 \dagger$ \\
\hline 2.54 vs. 12.70 & 14 of 15 & $93 \dagger$ & 15 of 15 & $100 \dagger$ & & \\
\hline 2.54 vs. 17.78 & 15 of 15 & $100 t$ & 15 of 15 & $100 t$ & & \\
\hline 5.08 vs. 7.62 & 10 of 15 & 67 & 12 of 14 & $86 t$ & & \\
\hline 5.08 vs. 10.16 & 13 of 15 & $87 t$ & 15 of 15 & $100 \dagger$ & & \\
\hline 5.08 vs. 12.70 & 15 of 15 & $100 \dagger$ & 15 of 15 & $100 \dagger$ & 12 of 12 & $100 t$ \\
\hline 5.08 vs. 17.78 & 14 of 15 & $93 \dagger$ & 15 of 15 & $100 \dagger$ & & \\
\hline 7.62 vs. 10.26 & 11 of 15 & 73 & 13 of 13 & $100+$ & & \\
\hline 7.62 vs. 12.70 & 14 of 15 & $93 \dagger$ & 15 of 15 & $100+$ & 10 of 12 & $83 \dagger$ \\
\hline 7.62 vs. 17.78 & 15 of 15 & $100 \dagger$ & 15 of 15 & $100 \dagger$ & 12 of 12 & $100 \dagger$ \\
\hline 7.62 vs. 22.86 & & & & & 11 of 12 & $92 \dagger$ \\
\hline 10.16 vs. 12.70 & 10 of 15 & 67 & 14 of 15 & $93 \dagger$ & & \\
\hline 10.16 vs. 17.78 & 15 of 15 & $100 \dagger$ & 14 of 15 & $93 \dagger$ & 12 of 12 & $100 \dagger$ \\
\hline 12.70 vs. 17.78 & 404 of 405 & $100 \dagger$ & 404 of 405 & $100 \dagger$ & 12 of 12 & $100 \dagger$ \\
\hline 12.70 vs. 22.86 & 14 of 15 & $93 t$ & 13 of 15 & $87 \dagger$ & 12 of 12 & $100 \dagger$ \\
\hline 12.70 vs. 27.94 & 12 of 15 & $80 \dagger$ & 6 of 15 & $40 \dagger$ & 12 of 12 & $100 \dagger$ \\
\hline 12.70 vs. 33.02 & 10 of 15 & 67 & 3 of 15 & $20 \dagger$ & & \\
\hline 15.24 vs. 27.94 & & & & & 12 of 12 & $100 \dagger$ \\
\hline 17.78 vs. 22.86 & 3 of 15 & $20 \dagger$ & 2 of 15 & $13 \dagger$ & 12 of 12 & $100 \dagger$ \\
\hline 17.78 vs. 27.94 & 2 of 15 & $13 \dagger$ & 4 of 15 & 27 & 12 of 12 & $100 \dagger$ \\
\hline 17.78 vs. 33.02 & 5 of 15 & 33 & 0 of 15 & $0+$ & 12 of 12 & $100 \dagger$ \\
\hline 20.32 vs. 27.94 & & & & & 12 of 12 & $100 t$ \\
\hline 22.86 vs. 27.44 & 10 of 15 & 67 & 5 of 15 & 33 & 296 of 312 & $95 \dagger$ \\
\hline 22.86 vs. 33.02 & 8 of 15 & 53 & 6 of 15 & 40 & 12 of 12 & $100 \dagger$ \\
\hline 27.94 vs. 33.02 & 5 of 15 & 33 & 8 of 15 & 53 & 9 of 12 & 75 \\
\hline 27.94 vs. 38.10 & & & & & 9 of 12 & 75 \\
\hline 27.94 vs. 43.18 & & & & & 11 of 12 & $92 \dagger$ \\
\hline 30.48 vs. 38.10 & 9 of 15 & 60 & 7 of 15 & 47 & 12 of 12 & $100 \dagger$ \\
\hline 33.02 vs. 38.10 & & & & & 8 of 12 & 67 \\
\hline 33.02 vs. 43.18 & 9 of 15 & 60 & 7 of 15 & 47 & 12 of 12 & $100 \dagger$ \\
\hline 38.10 vs. 43.18 & & & & & 9 of 12 & 75 \\
\hline 38.10 vs. 45.72 & 6 of 15 & 40 & 9 of 15 & 60 & 9 of 12 & 75 \\
\hline 43.18 vs. 48.26 & & & & & 8 of 12 & 67 \\
\hline 43.18 vs. 50.80 & 10 of 15 & 67 & 8 of 15 & 53 & 5 of 12 & 42 \\
\hline
\end{tabular}

*This subject balked persistently for some pairs of small test stimuli, producing less than 15 trials where indicated. $\quad \dagger p<.05$. 
that would be produced by random responding. As this does not approach statistical significance, they can be regarded as random choices.

In sharp contrast, responses of the naive monkey (Burt) were relational on both far and near tests on both sides of the continuum. This result is quite similar to that obtained by Harmon et al. (1982) for naive monkeys. The only exceptions for Burt occurred on the test pair closest to each end of the continuum, which elicited essentially random responding. (Burt began to respond persistently in terms of a position habit on the 33rd trial of the last test session and continued to do so, regardless of whether the training stimuli or the novel test stimuli were presented; therefore, the data for only the four valid test sessions are presented.)

\section{Discussion}

The reversals by Penny and Lilly on near size transposition tests show that their discriminations were based on absolute qualities of the stimuli. It is interesting that the statistically significant reversals occurred on these near tests rather than on far tests. The shape of the generalization gradients originally postulated by Spence (1937) predicted that reversals would occur on far tests rather than on near tests. However, the narrow generalization gradients determined empirically for pigeons on a wavelength discrimination by Honig (1962) predicted reversals on near tests, and such reversals were actually obtained from his subjects. Near test reversals were also obtained by Ehrenfreund (1952) from white rats tested on size transposition. Reversals on near tests, but not on far tests, can readily be predicted from gradients that produce a much higher net tendency to approach the positive training stimulus than any other stimulus. This would be a likely result when inhibitory tendencies, if any, were relatively constant for all stimuli.

The responses of Burt, the naive monkey, to the size transposition test problems differed greatly from those of the two who had previously been trained on brightness discrimination. He consistently chose the large stimulus over a range of sizes comparable to that employed by Harmon et al. (1982). This indicates that their results are replicable, even though single subject research lacks the regularizing effects of grouped data. However, a broader range was tested in this experiment, and the random choices Burt made for the test pairs at the ends of this range indicate that he was not responding to the relation between the stimuli. The abruptness of the decrement in relational responding indicates that it is not merely a loss of stimulus control. Rather, this change in response pattern for test pairs would be expected if Burt's responses, like those of Penny and Lilly, were based on generalization gradients. The difference between the other 2 monkeys and Burt could be that Burt's gradients have much less slope so that they meet only much farther out on the size continuum. The responses of the naive monkeys trained and tested in the same manner by Harmon et al. (1982) can also be accounted for by relatively broad generalization gradients.
Discrimination training on one dimension can narrow the generalization gradients obtained after discrimination training on another dimension (Thomas, Freeman, Svinicki, Burr, \& Lyons, 1970). This explains the difference between performance by Penny and Lilly, previously trained to discriminate brightness, and that of Burt or the monkeys trained to discriminate size by Harmon et al. (1982). This explanation should account for the difference in the sharpness of their generalization gradients on the size transposition test. The magnitude of this effects depends in part on the particular stimulus dimensions involved (Thomas, 1969). To account for the present data, the effect of brightness discrimination training on the generalization of a size discrimination must be substantial.

\section{REFERENCES}

Ackles, P. K., Zimmerman, R. R., Manning, M., \& Kazarian, C. (1974). Transposition in young monkeys and children. Perceptual \& Motor Skills, 39, 1291-1298.

Bagshaw, M. H., \& Pribram, K. H. (1965). Effect of amygdalectomy on transfer of training in monkeys. Journal of Comparative \& Physiological Psychology, 59, 118-121.

ButTer, C. M., \& Doehrman, S. R. (1968). Size discrimination and transposition in monkeys with striate and temporal lesions. Cortex, 4, 35-46.

EHRENFREUND, D. (1952). A study of the transposition gradient. Journal of Experimental Psychology, 43, 81-87.

Harmon, K., Strong, R., \& PASnak, R. (1982). Relational responses in tests of transposition with rhesus monkeys. Learning \& Motivation, 13, 495-504.

HoNIG, W. K. (1962). Prediction of preference, transposition, and transposition-reversal from the generalization gradient. Journal of $E x$ perimental Psychology, 64, 239-248.

REESE, H. W. (1968). The perception of stimulus relations: Discrimination learning and transposition. New York: Academic Press.

SCHWARTZ, B. (1984). Psychology of learning and behavior. New York: Norton.

Schwartzbaum, J. S., \& Pribram, K. H. (1960). The effects of amygdalectomy in monkeys on transposition along a brightness continuum. Journal of Comparative \& Physiological Psychology, 53, 396-399.

SPENCE, K. W. (1937). The differential response in animals to stimuli varying within a single dimension. Psychological Review, 44, 430-444.

Tномаs, D. R. (1969). The use of operant conditioning techniques to investigate perceptual processes in animals. In R. M.Gilbert \& N. S. Sutherland (Eds.), Animal discrimination learning (pp. 1-33). New York: Academic Press.

Thomas, D. R., Freeman, F., Svinicki, J. G., Burr, D. E. S., LYONS, J. (1970). Effects of extradimensional training on stimulus generalization. Journal of Experimental Psychology Monographs, 83 (1, Pt. 2).

Zimmerman, R. W. (1968). Transposition in the infant and adult rhesus monkey. Perceptual \& Motor Skills, 26, 871-877.

(Manuscript received for publication November 4, 1986.) 